\title{
Correction to: Innovative deep learning models for EEG-based vigilance detection
}

\author{
Souhir Khessiba ${ }^{1,2} \cdot$ Ahmed Ghazi Blaiech $^{1,3} \cdot$ Khaled Ben Khalifa ${ }^{1,3} \cdot$ Asma Ben Abdallah $^{1,4} \cdot$ \\ Mohamed Hédi Bedoui ${ }^{1}$
}

Published online: 18 June 2021

(C) Springer-Verlag London Ltd., part of Springer Nature 2021

\section{Correction to: Neural Computing and Applications (2021) 33:6921-6937 \\ https://doi.org/10.1007/s00521-020-05467-5}

As the corresponding author of this article, has forgot to add an affiliation to the first author "Souhir Khessiba". It is added as follows:Souhir Khessiba ${ }^{1,2}$, Ahmed Ghazi Blaiech $^{1,3, *}$, Khaled Ben Khalifa ${ }^{1,3}$, Asma Ben Abdallah ${ }^{1,4}$, Mohamed Hédi Bedoui ${ }^{1}$

${ }^{1}$ Laboratoire de Technologie et Imagerie Médicale, Faculté de Médecine de Monastir Université de Monastir 5019 Monastir, Tunisie
${ }^{2}$ ISITCom, Université de Sousse, 4011, Sousse, Tunisie

${ }^{3}$ Institut Supérieur des Sciences Appliquées et de Technologie de Sousse Université de Sousse 4003 Sousse, Tunisie

${ }^{4}$ Institut supérieur d'informatique et de Mathématiques, Université de Monastir, 5019 Monastir, Tunisie

Publisher's Note Springer Nature remains neutral with regard to jurisdictional claims in published maps and institutional affiliations.

The original article can be found online at https:// doi.org/10.1007/s00521-020-05467-5.

Ahmed Ghazi Blaiech

ahmedghazi.blaiech@issatso.u-sousse.tn

1 Laboratoire de Technologie et Imagerie MédicaleFaculté de Médecine de Monastir, Université de Monastir, 5019 Monastir, Tunisia

2 ISITCom, Université de Sousse, 4011 Sousse, Tunisia

3 Institut Supérieur des Sciences Appliquées et de Technologie de Sousse, Université de Sousse, 4003 Sousse, Tunisia

4 Institut Supérieur d'informatique et de Mathématiques, Université de Monastir, 5019 Monastir, Tunisia 